\title{
PRÁTICAS DE PUBLICAÇÃo E AVALIAÇÃO EM CIÊNCIAS SOCIAIS E HUMANIDADES: CONTRADIÇÕES E DESAFIOS
}

\author{
Raimundo Nonato Macedo dos Santos \\ Universidade Federal de Pernambuco, Centro de Artes e Comunicação, Departamento de \\ Ciência da Informação \\ rnmacedo@uol.com.br

\begin{abstract}
Alejandro Caballero-Rivero Universidade Federal de Pernambuco, Centro de Artes e Comunicação, Departamento de Ciência da Informação caballero.alecaba@gmail.com
\end{abstract} \\ Nancy Sánchez-Tarragó \\ Universidade Federal de Pernambuco, Centro de Artes e Comunicação, \\ Departamento de Ciência da Informação \\ nancita1973@gmail.com
}

\begin{abstract}
Resumo
Este trabalho discute aspectos sobre as peculiaridades das práticas epistêmicas das Ciências Sociais e Humanidades que exigem avaliações diferenciadas da sua produção científica como condição para julgar, com equidade, seu valor e lugar no contexto do sistema de Ciência e Tecnologia brasileiro. Baseado em uma pesquisa bibliográfica que se reflete também em resultados de estudos empíricos nacionais e internacionais realizados, reforça a discussão de que se faz necessário, urgente, um aprimoramento da "ciência da avaliação científica" com vistas a corrigir as deficiências dos indicadores métricos vigentes nos meios acadêmicos, bem como que tome em consideração configurações de poder no interior e entre as comunidades científicas do país. Enfatiza a importância de que se compreendam as diferenças das práticas epistêmicas no contexto das comunidades acadêmicas, particularmente, sobre o ponto de vista de limitar o uso acrítico e quase absoluto dos indicadores.
\end{abstract}

Palavras-chave: Produção Científica; Indicadores Métricos; Práticas de Publicação e Avaliação; Ciências Sociais e Humanidades; Contradições e Desafios.

\section{PUBLISHING AND EVALUATION IN SOCIAL SCIENCES AND HUMANITIES: CONTRADICTIONS AND CHALLENGES}

\section{Abstract}

This paper discusses aspects about the peculiarities of the epistemic practices of the Social Sciences and Humanities that require differentiated evaluations of their scientific production as a condition to judge, with fairness, their value and place in the context of the Brazilian Science and Technology system. Based on a bibliographical research that is also reflected in the results of national and international empirical studies carried out, it reinforces the discussion that an improvement of the "science of scientific evaluation" is urgently needed in order to correct the deficiencies of the metric indicators in force in as well as taking into account power settings within and between the scientific communities of the country. It emphasizes the importance of

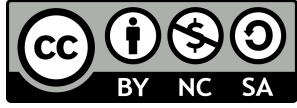

P2P \& INOVAÇÃO, Rio de Janeiro, v. 4 n. 1, p.18-34, Set./ Fev. 2018. 
understanding the temic practices in the context of academic communities, particularly from the point of view of limiting the uncritical and almost absolute use of indicators.

Key words: Scientific Production. Metric Indicators. Publication and Evaluation Practices. Social Sciences and Humanities. Contradictions and Challenges.

\section{Introdução}

Os estudos sobre a produção, circulação e avaliação do conhecimento científicos, desenvolvidos desde os anos de 1970, têm apontado para a existência de um sistema acadêmico mundial, com uma estrutura desigual, centrado na criação de um circuito de “corrente principal” que privilegia o artigo científico como canal de publicação. Por sua vez, os indicadores bibliométricos, fundamentalmente gerados por sistemas de indexação comercial como Web of Science, em primeiro lugar, e Scopus repercutem esse comportamento na forma de medidas da qualidade da produção científica mundial, assumidos, portanto, junto à comunidade científica como padrão de avaliação não só de revistas, mas também de pesquisadores e instituições.

Em torno desse circuito e suas bases de dados foi se forjando uma acumulação de capital científico e simbólico que vem beneficiando certas zonas geográficas, línguas e disciplinas e, paralelamente, ampliando a distância entre as áreas "periféricas" e aquelas com reconhecimento "internacional” (BEIGEL, 2013). Recorrentemente, as disciplinas beneficiadas, sem dúvida, são as incluídas dentro das Ciências Exatas, Naturais e as Engenharias, cujas práticas epistêmicas predominam e têm levado a um viés no gerenciamento dos sistemas de pesquisa e avaliação, como destacam Carvalho e Manoel (2006).

Segundo esses autores, para as Ciências Sociais e Humanas só restam dois caminhos: “ou desprezam a política científica ou abandonam suas convicções para adequar sua produção ao modelo vigente de modo a atender o que apontam os indicadores". É evidente que ambos os caminhos trazem impactos lesivos para a promoção, qualificação e avanço do conhecimento científico.

O presente trabalho, baseado em uma pesquisa bibliográfica, mas utilizando também dados empíricos de estudos nacionais e internacionais, reforça a discussão já começada por outros trabalhos de que as Ciências Sociais e Humanas brasileiras apresentam peculiaridades nas suas práticas epistêmicas que exigem avaliações diferenciadas da sua produção científica 
como condição para julgar com equidade seu valor e lugar dentro do sistema de ciência e tecnologia do país. Considera-se que essa é uma discussão que continua sendo pertinente e atual, embora de solução complexa.

O trabalho está estruturado em três partes. A primeira apresenta uma revisão de estudos nacionais e internacionais que estabelecem as principais diferenças entre as duas grandes culturas epistêmicas: as Ciências Exatas, Naturais e as Engenharias, e as Ciências Sociais e Humanas, no que diz respeito à frequência de publicação, veículos de publicação preferidos, extensão dos textos, língua e orientação nacional ou internacional dos veículos preferidos. A segunda parte revisa brevemente a avaliação científica, com ênfase no sistema de avaliação brasileiro. A terceira parte apresenta, em considerações finais, reflexões sobre as consequências da aplicação padronizada de indicadores quantitativos, sem levar em conta as diferenças disciplinares, assim como alguns dos caminhos alternativos.

\section{Diferenças entre culturas epistêmicas}

Os estudos de sociologia do conhecimento científico têm mostrado que cada domínio científico ou disciplina caracteriza-se por estruturas e processos de apropriação, geração e comunicação de conhecimento específicos. Essa perspectiva responde ao que Knorr-Cetina define como culturas epistêmicas, i.e., “[...] aquelas amálgamas de acordos e mecanismos [...] que, em um determinado campo, conformam como sabemos o que sabemos. As culturas epistêmicas são culturas que criam e justificam os conhecimentos [...]” (KNORR- CETINA, 1999, apud CRONIN, 2003, p.5). Fazer de um modo determinado uma pesquisa, escrever um texto científico, publicá-lo são algumas práticas epistêmicas inseridas em culturas disciplinares específicas.

O trabalho de Becher (1994) indica a existência de clusters intelectuais que permitem discernir traços característicos e diferenciadores associados aos objetos de estudo, métodos e recursos de pesquisa das Ciências Exatas, Naturais e Engenharias, e das Ciências Sociais e Humanas. Embora com contestações, tradicionalmente, as primeiras têm sido chamadas de ciências "duras" e as segundas, ciências "moles", com subclassificações como "puras" e “aplicadas" para cada categoria. Sem entrar no mérito da discussão dessa classificação, que, certamente, parece ser mais política que científica, pode-se afirmar que existem traços que diferenciam as comunidades referenciados a suas culturas epistêmicas. 
Conforme Meadows (1999), os métodos, as teorias, o equipamento e as regras que funcionam ao interior das comunidades acadêmicas são diferentes. As Ciências Exatas e da Natureza são menos flexíveis, as pesquisas são desenvolvidas sobre a base do paradigma dominante no campo; utilizam métodos mais quantitativos e rigorosos; necessitam de uma infraestrutura de pesquisa mais cara; obtêm maior financiamento para a pesquisa; e os pesquisadores precisam estar atualizados sobre os últimos resultados de pesquisa (não dos clássicos). Por sua vez, nas Ciências Sociais e Humanas realizam-se pesquisas com metodologias mais flexíveis, com um enfoque mais qualitativo, num contexto marcado por diversas correntes teóricas; os resultados não são considerados como universais, as pesquisas não precisam de uma infraestrutura cara, o financiamento é menor e o papel dos clássicos para os pesquisadores é essencial.

As diferenças antes mencionadas implicam disparidades nas práticas epistêmicas das áreas: tipos de veículos de publicação preferidos, extensão dos textos, frequência de publicação, orientação nacional ou internacional das publicações escolhidas, preferência de linguagem dos textos, entre outras.

Com relação à frequência de publicação e veículos preferidos, Meadows (1999) analisa o desempenho per capita dos pesquisadores nas universidades britânicas e expõe que, enquanto os das Ciências Naturais, Exatas, Tecnológicas e Médicas publicam 5,52 - 6,12 artigos e apenas 0,15 - 0,18 livros, a produção de seus pares das Ciências Sociais e Humanidades é de 2,30 - 2,73 artigos e 0,64 - 0,68 livros. Por outra parte, a pesquisa de Hicks (1999) indica que, na Austrália, os pesquisadores das Ciências Naturais publicam $85 \%$ da sua produção em periódicos ou em anais de eventos, enquanto que seus pares das Ciências Sociais e Humanidades publicam $61 \%$ nesses canais. Na Espanha, enquanto que as Ciências Naturais publicam $81 \%$ da sua produção em periódicos, as Ciências Sociais e Humanidades atingem 54\%. Na Alemanha, na área de Sociologia, os pesquisadores publicam $42 \% \mathrm{em}$ revistas. Finalmente, os filósofos holandeses publicam 60 - 66\% da sua produção em artigos.

Segundo o estudo de RIN (2009), também para os pesquisadores britânicos, os artigos em revistas constituem o canal de comunicação mais utilizado em todas as grandes áreas do conhecimento, incluindo as Ciências Humanas, Artes e Letras. Assim, nas Ciências Biológicas e da Saúde alcançam $\sim 70 \%$ da produção científica; nas Ciências Exatas e Naturais, 80\%; nas Engenharias, 50\%; nas Ciências Sociais, 70\%; nas Ciências Humanas, $\sim 55 \%$; e em Artes e Letras, $\sim 65 \%$. No entanto, as monografias atingem valores significativos nas Ciências Humanas ( 35\%); nas Ciências Sociais, 15\%; mas não nas Artes e Letras, 
25. Similarmente, o trabalho de Piro, Aksnes e Rørstad (2013) sobre os pesquisadores noruegueses identificou que as monografias predominam em mais do $60 \%$ das publicações nas Ciências Humanas e $50 \%$ nas Ciências Sociais; em contraste, desempenham um papel menor do que nas outras ciências.

Essas diferenças também se manifestam na extensão e na frequência de publicação, em correspondência com o tipo de veículo de comunicação. Os artigos são publicações mais curtas e sintéticas, possibilitando uma escritura e leitura mais rápidas; são publicados periodicamente, permitindo uma rápida atualização; sua disseminação é maior; portanto, respondem às necessidades das ciências "duras" que lidam, predominantemente, com fenômenos determinísticos e que produzem conhecimento a uma velocidade superior. Por sua vez, os livros são publicações mais longas, pelo que sua escrita, edição e leitura levam mais tempo; seu grau de atualização é menor; sua disseminação não atinge o nível dos artigos e são mais apropriados para a publicação de conhecimentos mais sedimentados. Esses elementos respondem melhor à natureza das Ciências Sociais e Humanidades, que lidam com fenômenos estocásticos e produzem um conhecimento mais complexo, exigindo um nível superior de elaboração e argumentação e, consequentemente, de mais tempo e espaço para a sua exposição.

Fry et al. (2009) verificaram que na Biologia os pesquisadores tipicamente publicam 1-2 artigos por ano de 4.000 a 6.000 palavras, com a pressão para produzir, ocasionalmente, uma monografia de 20.000 palavras. Nas Engenharias um pesquisador publica ao longo de sua carreira um total de 30 a 60 artigos de 4.000 a 6.000 palavras, no entanto, prioriza a produção adicional de relatórios e outros documentos técnicos. Na Química, os pesquisadores produzem anualmente 10 - 12 artigos com menos de 4.000 palavras. Na Economia são produzidos 2 - 4 artigos por ano de 8.000 palavras, mais livros. Na História e na Linguística os pesquisadores produzem anualmente um trabalho de 8.000 - 12.000 palavras e, ao mesmo tempo, trabalham num livro.

No que diz respeito à orientação nacional ou internacional das publicações, ela varia em conformidade com a disciplina, pois tem muito a ver com a linguagem dos textos, as características da construção do objeto científico e a relação da pesquisa com a realidade local ou regional. A linguagem, seja verbal ou mental, é precisa para observar e representar o mundo. Como escreveram Prigogine e Stengers (1979), apud Fourez (1995), os cientistas não são indivíduos observando o mundo com base em nada, são os participantes de um universo cultural e linguístico no qual inserem seus projetos individuais e coletivos. 
Ortiz (2004) analisa diferenças entre as Ciências Humanas e Sociais e as Ciências Exatas e da Natureza com respeito ao uso da linguagem, tanto como ferramenta instrumental, pragmática, como no sentido da estrutura dos discursos. No caso das Ciências Sociais e Humanas, linguagem e contexto se entrelaçam, sendo o pensamento nessas áreas uma tradução, algo intermediário entre o ideal de universalidade e o enraizamento dos fenômenos sociais, locais ou regionais. Nas Ciências Exatas e Naturais, no entanto, é possível depurar a linguagem de sua malha sociocultural e existem noções e códigos com maior consenso universal. Para as Ciências Humanas e Sociais, então, são maiores as dificuldades na hora de escrever ou traduzir seus textos em línguas diferentes das "nacionais". Retomando as palavras de Ortiz (2004), "a língua reforça o vínculo discursivo ao solo onde as Ciências Sociais são elaboradas". Como ele afirma, o problema não é só de encontrar termos e conceitos equivalentes, mas de expressar tradições, correntes de pensamento ou contextos históricos próprios e específicos de um país ou região. Por outra parte, a construção do objeto científico, seu recorte conceitual, acontece por meio da língua e seu poder argumentativo. Portanto, não é coincidência a escolha de determinado idioma, mas uma questão de grande importância na concepção final do discurso. Essa sujeição ao contexto e à linguagem compromete a internacionalização da produção científica das Ciências Sociais e Humanas, ainda mais, quando a internacionalização é concebida atualmente como escrever em inglês e publicar em revistas anglófonas (LILLIS; CURRY, 2010).

\subsection{As práticas de publicação em Ciências Sociais e Humanas no Brasil}

Dentre as práticas epistêmicas, as práticas de publicação têm um papel preponderante. Estudos sobre a produção científica brasileira mostram que, no país, as comunidades acadêmicas também manifestam preferências pelo uso de determinados veículos de publicação. Trzesniak (2012) identificou um perfil específico de produção das grandes áreas que representam as ciências "duras” (Ciências Exatas, Naturais, Engenharias, Computação, Biológicas e da Saúde) e outro perfil nas Humanidades (Ciências Sociais Aplicadas, Humanas, Linguística, Letras e Artes). Nas ciências “duras" manifesta-se uma clara preferência pela publicação de artigos em periódicos: a razão (números de artigos / número de capítulos e livros) é 5,99, i.e., essas grandes áreas publicam seis artigos para cada capítulo ou livro. Nas Humanidades existe um maior equilíbrio entre os dois tipos de publicações $(1,16)$. Adicionalmente, a produção em artigos nas ciências "duras" realiza-se, 
majoritariamente, nas revistas internacionais, enquanto que, nas Humanidades, a preferência é para periódicos nacionais.

Mais recentemente, a pesquisa de Caballero-Rivero (2017) analisou a produção científica dos pesquisadores doutores das grandes áreas do CNPq entre 2000 e 2014 em artigos, livros, capítulos de livros e trabalhos em anais de eventos e confirmou que os resultados não têm variado significativamente ao logo do tempo com respeito aos achados de Trzesniak, mencionados no parágrafo anterior, embora existam algumas mudanças e peculiaridades no interior de algumas áreas. Além disso, a pesquisa de Caballero-Rivero mostra que, no contexto brasileiro, os trabalhos em anais constituem um canal de comunicação expressivo em várias grandes áreas.

Com relação às Humanidades (Ciências Sociais Aplicadas, Humanas, Linguística, Letras e Artes) manifesta-se o equilíbrio não só entre a produção de artigos e monografias (livros e capítulos de livros), mas, também, com relação a trabalhos em anais (Gráfico 1). No entanto, os artigos e trabalhos em anais constituem as principais práticas de publicação. Os artigos predominaram, segundo os censos realizados pelo CNPq em 2000-2004 e 2014, contribuindo com 37\% - 40\% da produção científica; os trabalhos em anais dominaram os censos de 2006 - 2010 com 40\% - 42\% dessa produção. Por sua vez, identifica-se uma estabilidade na contribuição das monografias para a produção científica. Enquanto que a contribuição dos artigos e trabalhos em anais oscila ao longo do período analisado, as monografias alcançam resultados estáveis, atingindo 26 - 28\% da produção científica.

Gráfico 1 - Produção de artigos, monografias e trabalhos em anais - Humanidades (censos 2000 2014)

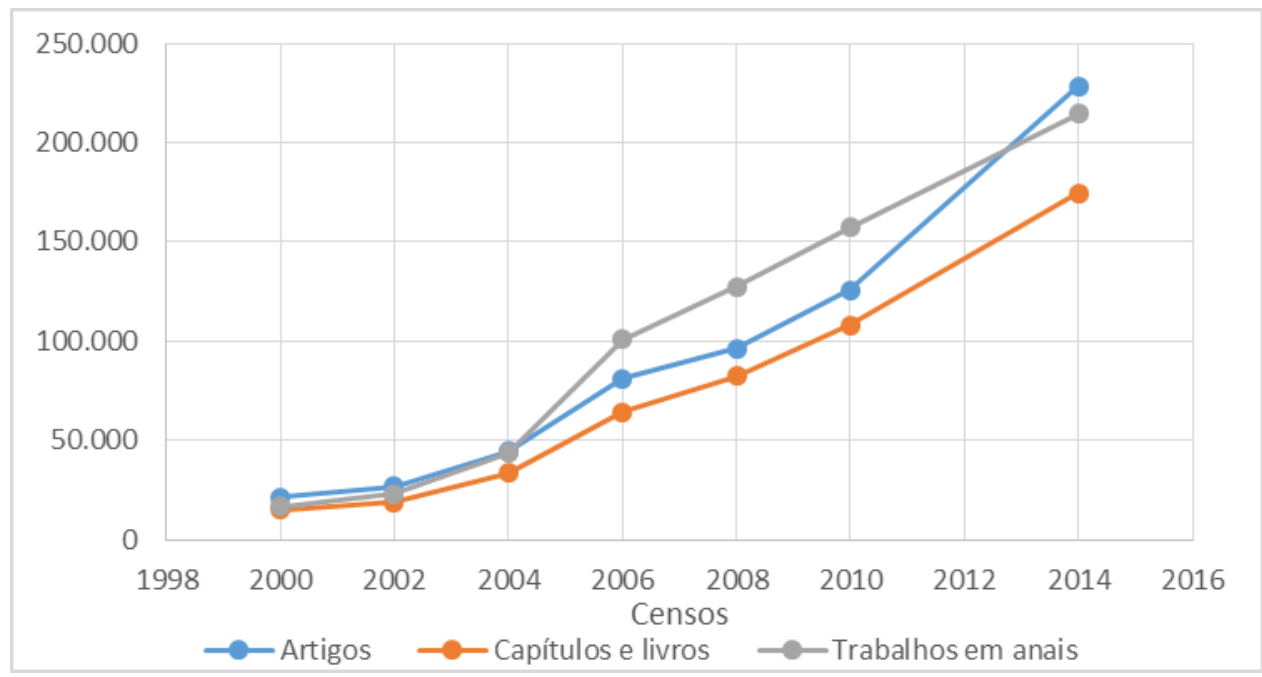

Fonte: Caballero-Rivero (2017) 
Por sua parte, Fukahori (2017) estudou as práticas de publicação dos bolsistas de produtividade PQ 1 e PQ-SR do CNPq das áreas da Ciência da Informação, Comunicação, Letras e Artes, no período de 1998 até 2013. O estudo também apontou a predominância da preferência de publicação através de artigos, bem como o incremento na produção de capítulos de livro. Nesse estudo, o livro apareceu como última preferência de publicação em todas as áreas analisadas. Assim, o autor constatou que este canal de publicação reconhecido tradicionalmente pela relevância nas ciências Humanas e Sociais aos poucos perde espaço para outros tipos de publicação, pelo menos nas áreas estudadas. O incremento dos capítulos de livros em detrimento do livro faz supor uma adaptação das práticas de publicação às exigências da produtividade, visto que, como o autor destaca, é mais fácil e requer menos esforço intelectual compor um livro com capítulos avulsos em forma de coletânea sobre um tema de autoria compartilhada do que escrever um livro em autoria única.

\section{Avaliação da produção científica no Brasil}

A importância da produção científica como fator que impulsiona a CT\&I é altamente reconhecida e, portanto, recebe uma atenção crescente. Sua avaliação é importante, uma vez que se faz necessário algum critério para alocar fundos e certificar-se de seu uso apropriado. No entanto, tal avaliação tem evidenciado dificuldades práticas. Um primeiro problema está vinculado à proliferação de indicadores quantitativos, os quais, muitas vezes, não têm outra justificativa clara para a sua escolha do que o fato de que os dados existem e, portanto, os indicadores podem ser gerados a partir deles (LANE; LARGENT; ROSEN, 2014). Vários autores nacionais e internacionais têm apontado as fragilidades dos indicadores quantitativos para avaliar uma noção tão relevante como a qualidade científica, seja aqueles que medem número de publicações, número de citações ou, inclusive, outros mais sofisticados que estabelecem a relação entre número de documentos publicados e número de citações como o fator de impacto (FI) e o índice H (CAMARGO Jr, 2010; 2013; DORA, 2012, SEGLEN, 1992).

Em um debate sobre a questão que referencia o item 3 acima, Souza (2013) argumenta:

A pretensão de avaliar a qualidade da produção científica por meio da relação entre número de documentos publicados e número de citações é prejudicada por fatores que os indicadores não podem captar, embora 
influenciem seus resultados: as diferenças da quantidade de documentos e da densidade de citações por área do conhecimento, os ritmos distintos de obsolescência das publicações, as variações de escopo entre as bases bibliográficas e ainda os diferentes mecanismos de buscas. (SOUZA, 2013, p. 2).

Um dos problemas antes apontados tem a ver com as fontes de dados utilizadas para a elaboração dos indicadores. As bases de dados eletrônicas multidisciplinares Web of Science (WoS), da Clarivate Analytics e Scopus da Elsevier, têm-se estabelecido como as alternativas mais utilizadas para a produção de indicadores, devido a que contam com uma ampla cobertura multidisciplinar; detalhados registros bibliográficos; identificação adequada dos coautores; disponibilidade de dados sobre as citações, produtos analíticos, entre outras características. No entanto, o escopo e cobertura das áreas de conhecimento é desigual. Assim, mostram uma cobertura mais abrangente nas ciências "duras" (Física, Química, Biologia molecular, Bioquímica, etc.), mas têm uma representação um pouco mais limitada no caso das Ciências Sociais e Humanidades, e naquelas disciplinas que lidam com problemas de pesquisa considerados como regionais ou nacionais (não universais) e que se apresentam de forma mais frequente nos países do hemisfério sul; por exemplo, a epidemiologia do vírus de imunodeficiência humana (VIH) na África subsaariana (MOED, 2007; HICKS; WANG, 2009; HICKS; WOUTERS, 2015). Também, as bases privilegiam a cobertura de artigos em detrimento de outros tipos de documentos como livros, capítulos de livros e anais de eventos.

Por demais, essas bases de dados têm permitido a construção de noções com alta carga geopolítica, como "centros de excelência", "revistas de corrente principal" e "universidades de classe mundial", a partir da aliança entre os sistemas de indexação bibliográfica e o campo editorial especializado, no qual a maioria das revistas "de corrente principal" são publicadas por oligopólios editoriais e de comunicação que operam detrás dos próprios Web of Science, Scopus, o diretório Ulrich, e outros. Esse sistema comporta, portanto, poucas revistas não anglófonas e não comerciais (SÁNCHEZ-TARRAGÓ; BUFREM; SANTOS, 2015).

No contexto brasileiro existem várias ferramentas para tentar avaliar a produção científica dos Programas de Pós-Graduação e dos pesquisadores: Qualis Periódicos (QP) para as publicações em artigos, o Roteiro para a Classificação de Livros (RCL), o Qualis Artístico (QA) para a produção artística (música, artes visuais, artes cênicas), e até 2009 existiu a qualificação de eventos (QE) (CAPES, 2015; CAPES, 2013; CAPES 2009). No entanto, a 
avaliação dos Comitês de Área da CAPES (CAs) e dos Comitês de Assessoramento do CNPq (CAS) manifesta um uso preponderante de Qualis Periódico (QP) como ferramenta de avaliação, em detrimento de outros mecanismos que permitam avaliar a produção científica comunicada em outros tipos de documentos. O Qualis Periódico é uma hierarquização dos periódicos que realizam os Comitês de Avaliação (CAs) dos Programas de Pós-Graduação da CAPES, a partir de diferentes critérios.

O levantamento realizado por Caballero-Rivero (2017) sobre os critérios utilizados pelos CAs, na Comissão Trienal 2013, para conformar o Qualis Periódico, mostra que o fator de impacto (FI) é utilizado por 35 dos 49 CAs (71\%) como critério principal para definir os estratos do QP, seja por meio de seu uso direito ou como alguma das suas variantes. O FI é utilizado predominantemente pelos CAs das ciências "duras" (31), mas também pelos CAs das Humanidades (4). As outras métricas mais utilizadas, embora em muito menor grau, são o Scimago Journal Ranking (SJR) por oito CAs (16\%); Cites per Do, por seis CAs (12\%) e o índice $\mathrm{H}$ por cinco CAs (10\%), as quais podem ser combinadas ou não com o FI. Esse enfoque apresenta algumas dificuldades, particularmente, que não é possível assumir que a qualidade de uma revista garanta necessariamente a qualidade dos artigos publicados nela.

Por outra parte, enquanto todos os CAs (49) avaliam a produção em artigos, só 33 (67\%) avaliam capítulos e livros; desses 33, 12 (36\%) somente aceitam livros se responderem a critérios específicos (livros com participação de docentes e discentes dos PPGs; livros vinculados às linhas de pesquisa dos PPGs; livros voltados para a educação básica, entre outros).

Outro levantamento realizado na mesma data, a partir dos documentos emitidos pelos Comitês de Assessoramento (CAS) do CNPq que estabelecem os critérios de julgamento para a concessão de bolsas de produtividade no período 2015-2017, mostra que, enquanto todos os CAS (50) exigem artigos publicados em periódicos, menos da metade (23 - 46\%) considera capítulos ou livros. Desses $23 \mathrm{CAS}$, apenas quatro (17\%) exigem que os pesquisadores tenham publicado capítulos ou livros, além de artigos; 13 (57\%) os consideram com o mesmo peso que os artigos; quatro (17\%) os consideram com menor peso; três (13\%) exigem que sejam publicados por editoras reconhecidas (grande circulação nacional ou internacional) e três $(13 \%)$ consideram capítulos ou livros apenas como critério de desempate entre pares (CABALLERO-RIVERO, 2017).

É possível apreciar que o uso do QP predomina, tanto na avaliação dos PPGs, quanto na avaliação dos pesquisadores. Semelhante abordagem é problemática; como afirmam 
Santos e Kobashi (2009, p. 159) “[...] não se pode reduzir a atividade científica à produção, à circulação e ao consumo de artigos de periódicos e, muito menos, confundir o crescimento quantitativo de artigos com o desenvolvimento cognitivo da ciência”.

No caso das Humanidades, o critério mais utilizado é a indexação de artigos em bases de dados reconhecidas internacionalmente, principalmente, as produzidas no hemisfério norte. Dos 17 CAs, 16 (94\%) utilizam SCOPUS, WoS, EBSCO, GALE, IBSS, Ulrich International Periodical Directory, PsycINFO, entre outras que pertencem a empresas privadas. Também são utilizadas algumas bases que não pertencem a empresas privadas, como DOAJ, ERIH Plus, The Philosopher's Index, The International Philosophical Bibliography, Francis-Bulletin Signaletique, American Theological Library Association (ATLAS), HAPI, e algumas do hemisfério sul, como SciELO, Latindex e Redalyc.

Tanto no caso dos CAs das ciências "duras", quanto das Humanidades, utilizam-se, majoritariamente, indicadores e critérios de revistas indexadas em bases de dados do hemisfério norte (fundamentalmente WoS). Trata-se de publicações em língua inglesa e que focam na ciência de interesse dos países desenvolvidos. São vieses que impactam negativamente, tanto na quantidade de periódicos brasileiros indexados nessas bases, quanto nos domínios do conhecimento, áreas e linhas de pesquisa de interesse para o país, i.e., a relevância social dos trabalhos deve ceder seu lugar ao interesse das revistas onde os trabalhos serão publicados.

\section{Considerações finais: contradições e desafios}

Considerando que os estudos cienciométricos e bibliométricos, sem sombra de dúvida, são altamente sensíveis ao tipo de documento que se assume como unidade de análise e à interpretação desses resultados, o uso exclusivo dos artigos para analisar a produção científica, submetido a essa perspectiva, deixa de considerar as práticas de publicação específicas que se manifestam no interior das diferentes comunidades acadêmicas. Assim, estudos com essas características podem ser representativos da produção científica das comunidades que conferem uma importância maior à publicação de artigos, mas não das Ciências Sociais e Humanidades, nas quais manifesta-se um equilíbrio entre os artigos e outros canais de comunicação.

Por outra parte, os critérios de avaliação que envolvem financiamento ou prestígio influenciam o comportamento dos pesquisadores em duas formas. Primeiro, quando os 
investigadores passam a considerar a obtenção de bons resultados nas suas avaliações como um fim que precisam atingir a qualquer preço (CCA, 2012). Nesse caso, o vínculo de reputação/financiamento com o número de publicações pode incentivar os pesquisadores a publicarem mais, em detrimento da qualidade (GODOI; XAVIER, 2012; BUTLER, 2003). Segundo, quando induzidos pelos critérios de avaliação, os pesquisadores introduzem mudanças nas práticas de publicação, particularmente, uma concentração progressiva em artigos de revistas (BUTLER, 2007).

O exemplo típico é o que vem acontecendo nas áreas de Ciências Sociais e Humanidades: independentemente de que no interior dessas comunidades a publicação de livros ou capítulos de livros tradicionalmente tenha trazido reconhecimento dos pares, a falta de indicadores de avaliação influi para que estes não sejam ponderados adequadamente. Boero (2015) acresce que ainda quando os indicadores são considerados para avaliação, quase sempre recebem uma recompensa limitada; apesar de as monografias implicarem um esforço maior, são consideradas apenas unitariamente (semelhantemente aos artigos) e, muitas vezes, sua pontuação é menor. O que se observa, então, é uma tendência a mudar práticas epistêmicas, privilegiando a produção de artigos, que é mais "rentável”.

Pior ainda é que as mudanças nas práticas de publicação podem vir junto à mudança na própria construção do objeto científico. Desse modo, deslegitimam-se aquelas práticas que não estão orientadas a gerar "produtos" e tecnologias à imagem e semelhança das ciências duras, favorecendo a desvinculação do trabalho intelectual da reflexão ética e política.

Nessa perspectiva, é possível afirmar que os indicadores para a avaliação de pesquisadores e instituições têm a capacidade de mudar a dinâmica da produção científica, conforme as prioridades e os objetivos das políticas. Por sua vez, as comunidades acadêmicas respondem com mudanças que podem resultar em efeitos indesejados, seja tornando os resultados das suas avaliações como o objetivo essencial a ser atingido ou mudando suas prioridades de pesquisa e suas práticas epistêmicas (WHITLEY, 2011).

Por outra parte, é importante destacar que essas métricas medem apenas as publicações e suas citações, não o impacto das ciências sobre o mundo real. Como avaliar, então, aquelas atividades científicas que não resultam diretamente em produtos como artigos ou livros, mas em intervenções sociais ou outros benefícios associados à vida das pessoas?

No caso de Brasil, o uso quase exclusivo das bases do Web of Science e suas métricas de impacto, fundamentalmente o fator de impacto, para a conformação do Qualis Periódico exclui e desqualifica a grande maioria das revistas brasileiras, compromete a inclusão dos 
periódicos brasileiros nesses estratos e estabelece a submissão da ciência nacional aos ditames de empresas privadas do mundo desenvolvido. Adicionalmente, deixa-se de considerar a maior parte da produção brasileira em Humanidades, pois a publicação de artigos internacionais nessas áreas atinge apenas $5.1 \%$ da sua produção total (TRZESNIAK, 2012).

A aplicação desses indicadores de avaliação impacta nas práticas de publicação da comunidade acadêmica brasileira. Pelo fato de que uma quantidade maior de artigos possibilita aos PPGs obter uma pontuação superior, bem como de que os artigos são um tipo de publicação que exige menos fôlego do que os capítulos ou livros, é lógico que se manifeste uma tendência a uma produção maior de artigos. Como efeito nefasto, promove-se, assim, o "produtivismo", fenômeno resultante da avaliação quantitativa de publicações como base para a progressão acadêmica, i.e., a quantidade de publicações se torna mais importante do que a qualidade, impacto ou relevância para a sociedade (GODOI; XAVIER, 2012). Segundo essa perspectiva, concorda-se com Rosa (2008) quando afirma que a possibilidade dos PPGs de ascender nos rankings de classificação (do conceito 3 ao 7) e dos bolsistas de produtividade (do nível 2 ao 1A) é mais influenciada pela quantidade do que pela qualidade das publicações.

Apesar destas considerações, não restam dúvidas das complexidades do estabelecimento de um sistema da avaliação da qualidade da produção científica que leve em consideração os aspectos analisados. Num debate publicado pela Revista Cadernos de Saúde Pública em 2013, a raiz da crítica de Camargo Jr. (2013) ao uso dos indicadores quantitativos para a avaliação, várias propostas se apresentaram: julgamento por meio de uma comissão de pares, de cinco produtos escolhidos pelos próprios pesquisadores ou instituições que se submetem à avaliação; a diversificação das bases bibliográficas utilizadas para calculá-los e a utilização de indicadores atribuídos aos artigos individualmente e não às revistas nas quais foram publicados.

Cada uma das propostas tem seus prós e contras. Por uma parte, como garantir que as comissões julgadoras não sejam contaminadas por pressões lobistas ou corruptoras, por jogos de poder e preconceitos? Por outra, como prevenir que as métricas não sejam impostas por grupos com mais poder simbólico? Adicionalmente, como usar eficientemente outras bases de dados que não tenham ainda apurados seus mecanismos de normalização, depuração e recuperação de dados? 
Provavelmente, a melhor opção segue sendo uma combinação entre indicadores quantitativos e qualitativos. Como acertadamente aponta Barreto (2013), seria necessário um aprimoramento da "ciência da avaliação científica" que tente corrigir as deficiências dos indicadores bibliométricos em uso atualmente, além de entender como se configuram as relações de poder no interior e entre as comunidades científicas no Brasil. Precisa-se também compreender as diferenças das práticas epistêmicas dentro das comunidades científicas e a necessidade de limitar o uso acrítico e quase exclusivo desses indicadores.

\section{REFERÊNCIAS}

BARRETO, ML. Como avaliar as ciências com uma deficiente ciência da avaliação científica? Cad. Saúde Pública, v. 29, n. 9, p. 1707-1730, 2013. http://dx.doi.org/10.1590/ 0102-311X00115413

BECHER, T. The significance of disciplinary differences. Studies in Higher Education, v. 10, n. 2, 1994, p. 151-161. Disponível em: <http://srhe.tandfonline.com/doi/abs/10.1080/ 03075079412331382007>. Acesso: 18 nov. 2016.

BEIGEL, F. Centros y periferias en la circulación internacional del conocimiento. Nueva Sociedad, n. 245, p. 110-123. Disponível em: <http://nuso.org/articulo/centros-y-periferiasen-la-circulacion-internacional-del-conocimiento/

BOERO, F. We need monographs, and revisions. Italian Journal of Zoology, v. 82, n. 2, 2015, p. 149-150. Disponível em: <http://www.tandfonline.com/doi/pdf/10.1080/ 11250003.2015.1041718>.

BUTLER, L. Explaining Australia's increases share of ISI publications - the effects of a funding formula based on publication counts. Research Policy, v. 32, n. 1, p. 143-155, 2003. Disponível em: <http://committees.uwa.edu.au/acaboard/research/minutes/2005/ 10th_february_2005/rc2.pdf>. Acesso: 23 set. 2016.

BUTLER, L. Assessing university research: a plea for a balanced approach. Science and Public Policy, v. 34, n. 8, 2007. p. 567-574. Disponível em: $<$ https://academic.oup.com/spp/article-abstract/34/8/565/1662777/Assessing-universityresearch-A-plea-for-a?redirectedFrom=PDF>. Acesso: 30 mar. 2017.

BUTLER, L.; VISSER, M. J. Extending citation analysis to non-source items. Scientometrics, v. 66, n. 2, 2006, p. 327-343. Disponível em: <https://link.springer.com/ article/10.1007/s11192-006-0024-1>. Acesso: 23 fev. 2017.

CABALlERO-RIVERO, A. Caracterização das práticas de publicação das grandes áreas do conhecimento no Brasil. Dissertação (Mestrado em Ciência da Informação) - 
Programa de Pós-Graduação em Ciência da Informação, Universidade Federal de Pernambuco. Recife, 2017.

CAMARGO JR, K. R. Produção científica: avaliação da qualidade ou ficção contábil? Cad. Saúde Pública, v. 29, n. 9, p. 1707-1730, 2013. Disponível em: <http://dx.doi.org/10.1590/0102-311X00115413>.

CAMARGO JR, K. R. O rei está nu, mas segue impávido: os abusos da bibliometria na avaliação da ciência. Saúde \& Transformação Social, n. 1, p. 3-8, 2010.

CARVALHO, Y.M; MANOEL, E.J. Para além dos indicadores de avaliação da produção intelectual na grande área da saúde. Movimento, v.12, n. 03, p. 193-225, 2006.

CAPES - COORDENAÇÃO DE APERFEIÇOAMENTO DE PESSOAL DE NÍVEL SUPERIOR. Roteiro para classificação de livros, 2009. Disponível em: <https://www.capes.gov.br/images/stories/download/avaliacao/Roteiro_livros_Trienio2007_2 009.pdf>. Acesso: 18 mar. 2017.

CAPES - COORDENAÇÃO DE APERFEIÇOAMENTO DE PESSOAL DE NÍVEL SUPERIOR. Comunicado No 002/2013- Área de Artes - Música, 2013. Disponível em: <https://www.capes.gov.br/images/Comunicado_02-2013_atualiza\%C3\%A7\%C3\%A3o _QUALIS_Artistico_ref_2011_11_arte.pdf >. Acesso: 18 mar. 2017.

CAPES - COORDENAÇÃO DE APERFEIÇÕAMENTO DE PESSOAL DE NÍVEL SUPERIOR. Qualis. CAPES, 2015. Publicado: 29 nov. 2014. Última atualização: 22 jun. 2015. Disponível em: <http://www.capes.gov.br/acessoainformacao/perguntasfrequentes/avaliacao-da-pos-graduacao/7422-qualis>. Acesso: 16 mar. 2017.

CCA - COUNCIL OF CANADIAN ACADEMIES. Informing Research Choices: indicators and judgment. Otawa: Council of Canadian Academies, 2012. Disponível em: $<$ http://www.scienceadvice.ca/uploads/eng/assessments\%20and\%20publications $\% 20$ and $\% 20$ news \%20releases/science\%20performance/scienceperformance_fullreport_en_web.pdf $>$.

Acesso: 16 nov. 2016.

CRONIN, B. Scholarly Communication and Epistemic Cultures. Keynote Address, Scholarly Tribes and Tribulations: How Tradition and Technology Are Driving Disciplinary Change. ARL, Washington, DC, October 17, 2003. Disponível em: $<$ http://arl.nonprofitsoapbox.com/storage/documents/publications/scholarly-tribes-cronin17oct03.pdf>. Acesso: 14 jan. 2016.

DORA. San Francisco Declaration on Research Assessment. 2012. Disponível em: <http://am.ascb.org/dora/>. Acesso: 4 mar. 2017.

FOUREZ, G. A construção das ciências. Introdução a filosofia e ética das ciências. São Paulo: UNESP, 1995

FRY, J. et al. Communicating knowledge: how and why researchers publish and disseminate their findings. Disponível em: <http://www.jisc.ac.uk/media/documents/ publications/communicatingknowledge_focusgroupreport.pdf $>$. 
FUKAHORI, M.A.V. As práticas de publicação científica das áreas de ciências humanas e sociais aplicadas: investigação das preferências dos bolsistas de produtividade em pesquisa PQ1 e PQ-sr do CNPq. 2017. 81f. Dissertação (Mestrado em Ciência da Informação) Programa de Pós-Graduação em Ciência da Informação, Universidade Federal de Pernambuco. Recife, 2017.

GODOI, C. K.; XAVIER, W. G. O produtivismo e suas anomalías. Cad. EBAPE.BR, v. 10, n. 2, opinião 1, Rio de Janeiro, Jun. 2012. Disponível em: <http://www.scielo.br/pdf/cebape/v10n2/v10n2a12>. Acesso: 18 mar. 2017.

HICKS, D. The difficulty of achieving full coverage of international social Science literature and the bibliometric consequences. Scientometrics, Vol. 44, No. 2, pp. 193-215, 1999. Disponível em: <https://link.springer.com/article/10.1007/BF02457380>. Acesso: 18 mar. 2017.

HICKS, D; WANG, J. Towards a Bibliometric Database for the Social Sciences and Humanities. School of School of Public Policy, Georgia Institute of Technology, April 2009. Disponível em: <http://citeseerx.ist.psu.edu/viewdoc/download?doi=10.1.1.211.1429\& rep=rep1\&type=pdf $>$. Acesso em: 20 fev. 2016.

HICKS, D.; WOUTERS, P. The Leiden Manifesto for research metrics. Nature, Vol. 520, pp. 429-431, April 2015. Disponível em: <http://www.nature.com/news/bibliometrics-the-leidenmanifesto-for-research-metrics-1.17351>. Acesso em: 4 mar. 2017.

KNORR-CETINA, K. Epistemic cultures. How the sciences make knowledge. Harvard University Press, Cambridge Massachusetts, London, England, 1999.

LANE, J; LARGENT, M; ROSEN, R. Science Metrics and Science Policy. In: B. Cronin \& C. Sugimoto (Eds.), Beyond bibliometrics: Harnessing multidimensional indicators of scholarly impact (pp. 397-411). Cambridge, MA: MIT Press, 2014.

LILLIS, T.; CURRY, M. J. Academic writing in a global context. Routledge: New York, 2010. Disponível em: <https://www.warner.rochester.edu/files/news/files/ curry_book_10.pdf>. Acesso em: 30 jun. 2017.

MEADOWS, A, J. A comunicação científica. Brasília, DF: Briquet de Lemus Livros, 1999.

MOED, H. F. The future of research evaluation rests with an intelligent combination of advanced metrics and transparent peer review. Science and Public Policy, 34(8), October 2007, pages 575-583. Disponível em: <http://spp.oxfordjournals.org/content/34/8/ 575.full.pdf>. Acesso em: 14 jan. 2016.

ORTIZ, R. As Ciências Sociais e o Inglês. Revista Brasileira de Ciências Sociais, Vol. 19, n. 54, 2004. p. 5-23.

PIRO, F. N; ASKNESS, D. W.; RØRSTAD, K. A macro analysis of productivity differences across fields: challenges in the measurement of scientific publishing. Journal of the American Society for Information Science and Technology, v. 64, n. 2, 2013, p. 307-320. 
Disponível em: <http://onlinelibrary.wiley.com/doi/10.1002/asi.22746/abstract>. Acesso: 20 mar. 2017.

PRIGOGINE, I.; STENGERS, I. La nouvelle Alliance - Métamorphose de la science. Paris: Gallimard, 1979.

RIN - RESEARCH INFORMATION NETWORK. Communicating knowledge: how and why UK researchers publish and disseminate their findings. RIN Report, London: RIN, September, 2009. Disponível em: <http://rinarchive.jisc-collections.ac.uk/system/files/ attachments/Communicating-knowledge-report.pdf>. Acesso em: 20 mar. 2017.

ROSA, A. R. "Nós e os índices" - um outro olhar sobre a pressão institucional por publicação. RAE, Vol. 48, No. 4, pp. 108-114, 2008. Disponível em: <http://www.scielo.br/scielo.php?script=sci_arttext\&pid=S0034-75902008000400010>. Acesso em: 14. Mar. 2017.

SÁNCHEZ-TARRAGÓ, N.; BUFREM, L.S; SANTOS, R. N. M. La producción científica latinoamericana desde una mirada poscolonial. Tendências da Pesquisa Brasileira em Ciência da Informação., v.8, n.2, p.182-202, 2015. Disponivel em: http://inseer.ibict.br/ancib/index.php/tpbci/article/view/205 Acesso em: 4 jul. 2017.

SANTOS, R. N. M. dos; KOBASHI, N. Y. Bibliometria, cientometria, informetria: conceitos e aplicações. Pesq. bras. Ci. Inf., Brasília, v.2, n.1, p.155-172, jan./dez. 2009. Disponível em: <http://repositorio.ufpe.br/bitstream/handle/123456789/10089/BIBLIOMETRIA,\% 20CIENTOMETRIA,\%20INFOMETRIA_CONCEITOS\%20E\%20APLICA\%C3\%87\%C3\% 95ES.pdf? sequence=1\&isAllowed=y> . Acesso em: 2 mar. 2017.

SEGLEN, P.O. The skewness of science. J Am Soc Inf Sci, V. 43, p. 628-38, 1992.

SOUZA, L.E.P.F. O desafio da avaliação da produção científica. Cad. Saúde Pública, Rio de Janeiro, 29(9):1707-1730, set, 2013. Disponivel em: http://dx.doi.org/10.1590/0102311XCO050913 Acesso: 4 mar. 2017.

TRZESNIAK, P. A questão do livre acesso aos artigos publicados em periódicos científicos. Em Aberto, Brasília, v. 25, n. 87, p. 77-112, jan./jun. 2012. Disponível em: <http://emaberto.inep.gov.br/index.php/emaberto/article/view/2364/2326>. Acesso: 18 mar. 2017.

WHITLEY, R. Changing governance and authority relations in the public sciences. Minerva, v. $49, \quad$ n. $4, \quad$ p. 359-385, 2011. Disponível em: <https://link.springer.com/article/10.1007/s11024-011-9182-2>. Acesso. 23 out. 2016. 